\section{Endoscopic ultrasound-guided fine needle aspira- tion of splenic vein thrombosis: a novel approach to the portal venous system}
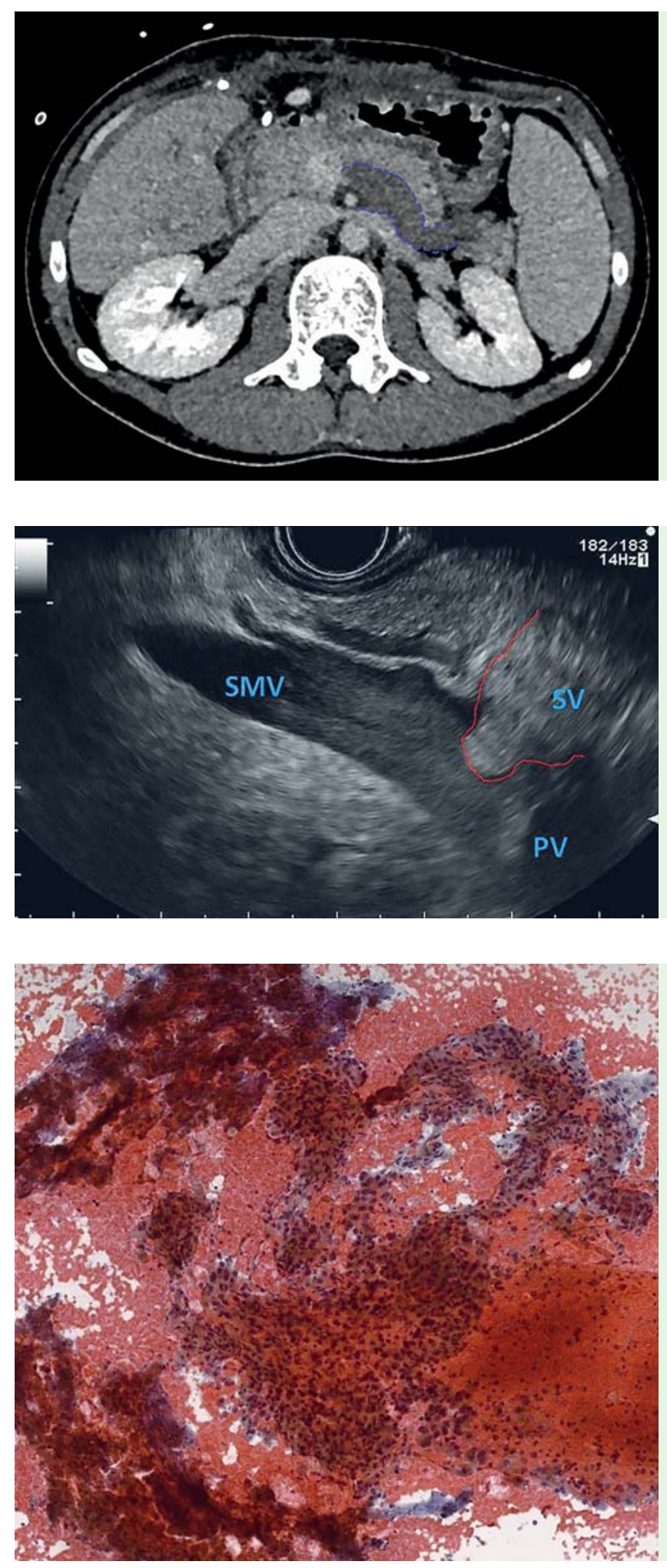

Fig. 1 Contrastenhanced computed tomography (CT) image showing a thrombus in the splenic vein.

Fig. 2 Endoscopic ultrasound (EUS) appearance of the portal vein confluence with a thrombotic mass floating into the confluence (red line). SMV, superior mesenteric vein; PV, portal vein; SV, splenic vein.

Fig. 3 Cytological appearance of the endoscopic ultrasoundguided fine needle aspiration (EUS-FNA) specimen showing pleomorphic liver cells with round nuclei and ample granular cytoplasm, diagnostic of hepatocellular carcinoma.
A diagnosis of portal system thrombosis can be easily obtained with conventional digital imaging [1]; however, the etiology of the thrombosis can be difficult to assess in the absence of the characteristic hallmarks of a malignant or benign thrombus. When a definitive diagnosis is required, transabdominal ultrasound-guided fine needle aspiration (FNA) of the portal vein thrombus can be performed [2], but this technique is not widely used because of the risk of post-biopsy bleeding complications.

We report the case of a 29-year-old man with a history of hepatocellular carcinoma (HCC), for which he had undergone a major hepatic resection when still a child. After a 20-year recurrence-free interval, a left lobe hepatectomy was performed because of HCC recurrence. The right lobe was treated 9 months later with multiple transarterial chemoembolizations because of multifocal recurrence. When, 6 months later, a major radiological and alpha-fetoprotein tumor response was observed, the patient underwent liver transplantation.

One year after the transplant, a splenic vein thrombosis was detected on a computed tomography (CT) scan ( $\bullet$ Fig. 1). An endoscopic ultrasound (EUS)-guided approach was considered the most appropriate to obtain a diagnosis as to the nature of the thrombus. An EUS-guided FNA was performed using a transgastric approach ( $\bullet$ Fig.2), with a 25-gauge needle (Wilson-Cook Medical, Winston-Salem, North Carolina, USA), without any immediate or delayed complications ( $\bullet$ Video 1 ).

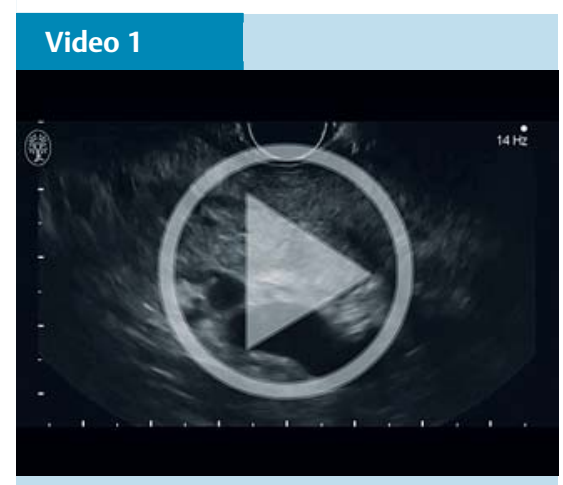

Endoscopic ultrasound (EUS) showing a complete thrombosis of the splenic vein from the splenic hilum to the portal confluence. A 25-gauge needle is advanced through the gastric wall and a thin portion of pancreatic parenchyma, into the splenic vein. Suction is applied and an aspirate obtained to be processed for cytology. 
Cytopathologic examination of the specimen revealed malignant cells consistent with HCC ( $\bullet$ Fig.3). Given the advanced stage of the disease, the patient was referred for systemic treatment. He died 9 months later.

EUS provides a unique opportunity to image the region in close proximity to the portal vein system. To our knowledge, only three cases of EUS-FNA of the portal vein have been reported $[3,4]$. This is the first reported case of EUS-FNA of the splenic vein.

When liver transplantation or major surgery is planned, a firm diagnosis of possible neoplastic invasion of the portal system is critical as macrovascular invasion is a major determinant of prognosis [5]. Conversely, EUS-FNA of a thrombus in the portal system may help to indicate interventional vs. systemic anti-thrombotic approaches in benign thrombosis. Future studies should establish the efficacy and safety profile of this technique, especially when vessel occlusion is partial or the thrombus is floating.

Endoscopy_UCTN_Code_TTT_1AS_2AC

Competing interests: None
Gabriele Delconte ${ }^{1,2}$, Sherrie Bhoori ${ }^{3}$, Massimo Milione ${ }^{4}$, Flaminia Cavallaro ${ }^{1}$, Enzo Masci ${ }^{1}$, Peter D. Siersema ${ }^{5}$, Vincenzo Mazzaferro ${ }^{3}$

${ }^{1}$ Diagnostic and Therapeutic Endoscopy Unit, Fondazione IRCCS Istituto Nazionale Tumori, Milan, Italy

2 Department of Oncology (DIPO), University of Milan, Milan, Italy

${ }^{3}$ Department of Surgery, Fondazione IRCCS Istituto Nazionale Tumori, Milan, Italy

${ }^{4}$ Department of Pathology, Fondazione IRCCS Istituto Nazionale Tumori, Milan, Italy

${ }^{5}$ Department of Gastroenterology and Hepatology, Radboud University Medical Center, Nijmegen, The Netherlands

\section{References}

1 Bach AM, Hann LE, Brown KT et al. Portal vein evaluation with US: comparison to angiography combined with CT arterial portography. Radiology 1996; 201: 149-154

2 Dodd GD 3rd, Carr BI. Percutaneous biopsy of portal vein thrombus: a new staging technique for hepatocellular carcinoma. AJR Am J Roentgenol 1993; 161: 229-233

3 Lai R, Stephens V, Bardales R. Diagnosis and staging of hepatocellular carcinoma by EUS-
FNA of a portal vein thrombus. Gastrointest Endosc 2004; 59: 574-577

4 Storch I, Gomez C, Contreras F et al. Hepatocellular carcinoma (HCC) with portal vein invasion, masquerading as pancreatic mass, diagnosed by endoscopic ultrasound-guided fine needle aspiration (EUS-FNA). Dig Dis Sci 2007; 52: 789-791

5 Minagawa M, Makuuchi M, Takayama Tet al Selection criteria for hepatectomy in patients with hepatocellular carcinoma and portal vein tumor thrombus. Ann Surg 2001; 233: 379-384

\section{Bibliography}

DOI http://dx.doi.org/

10.1055/s-0042-100199

Endoscopy 2016; 48: E40-E41

(c) Georg Thieme Verlag KG

Stuttgart · New York

ISSN 0013-726X

\section{Corresponding author}

\section{Gabriele Delconte, MD}

Diagnostic and Therapeutic Endoscopy Unit, Department of Surgery

Fondazione IRCCS Istituto Nazionale Tumori

Via G. Venezian 1

20133, Milan

Italy

gabriele.delconte@istitutotumori.mi.it 